\title{
Design and In-situ Synthesis of Titanium \\ Carbide/Boron Nitride Nanocomposite: Investigation of Electrocatalytic Activity for the Sulfadiazine Sensor
}

\author{
Thangavelu Kokulnathan ${ }^{\$}$, Elumalai Ashok Kumar ${ }^{\$}$, Tzyy-Jiann Wang ${ }^{\$ *}$ \\ ${ }^{\$}$ Department of Electro-Optical Engineering, National Taipei University of Technology, Taipei \\ 10608, Taiwan. \\ * Corresponding Author \\ E-mail: $\underline{\text { 10939@ntut.edu.tw }}$
}

Supporting Information content

Number of pages $\quad 3$

Number of figures $\quad 2$ 


\section{Chemicals}

Titanium aluminum carbide (TAC), ethanol, sulfadiazine (SFZ), hydrofluoric acid (HF), dimethyl sulfoxide (DMSO), boron nitride (BN), disodium phosphate, monosodium phosphate, and all other reagents were purchased from Sigma-Aldrich (Taipei, Taiwan). The phosphate buffer (PB) solution was used as an electrolyte for all sensing measurements.

\section{Characterizations}

The observation of nanocomposite images was conducted on a field emission scanning electron microscope (FESEM, JEOL 6500F). The nanocomposite characterization by transmission electron microscope (TEM, JEOL JEM-1200) includes high-resolution transmission electron microscopy (HRTEM), high-angle annular dark-field (HAADF) imaging-scanning transmission electron microscopy (STEM), fast Fourier transform (FFT), selected area electron diffraction (SAED), elemental mapping and energy dispersive X-ray (EDX) spectroscopy. X-ray diffraction (XRD) patterns were collected with an X-ray diffractometer with $\mathrm{Cu} K \alpha$ radiation (PANalytical B.V.). The electrochemical measurement (cyclic voltammetry and differential pulse voltammetry) was conducted on an electrochemical workstation ( $\mathrm{CH}$ Instruments) with a typical three-electrode setup, in which the glassy carbon electrode (GCE; $0.07 \mathrm{~cm}^{2}$ ), the silver/silver chloride (saturated potassium chloride) and the platinum wire were used as the working electrode, the reference electrode and the counter electrode, respectively.

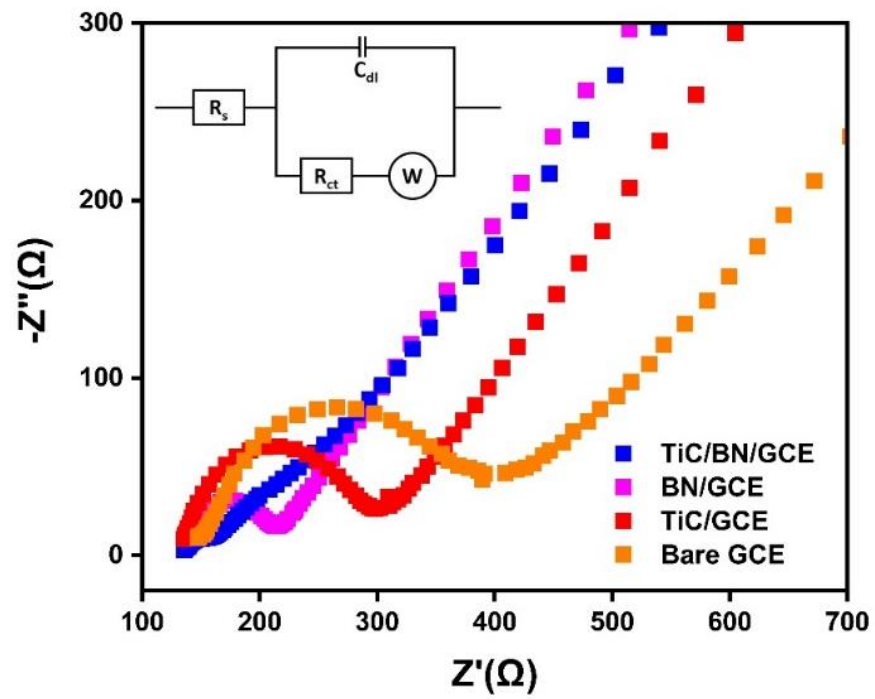

Fig. S1 EIS results obtained in $5 \mathrm{mM}\left[\mathrm{Fe}(\mathrm{CN})_{6}\right]^{3-/ 4-}$ containing $0.1 \mathrm{M} \mathrm{KCl}$ within a frequency range from $0.01 \mathrm{~Hz}$ to $100 \mathrm{kHz}$. (Inset: The equivalent circuit model). 


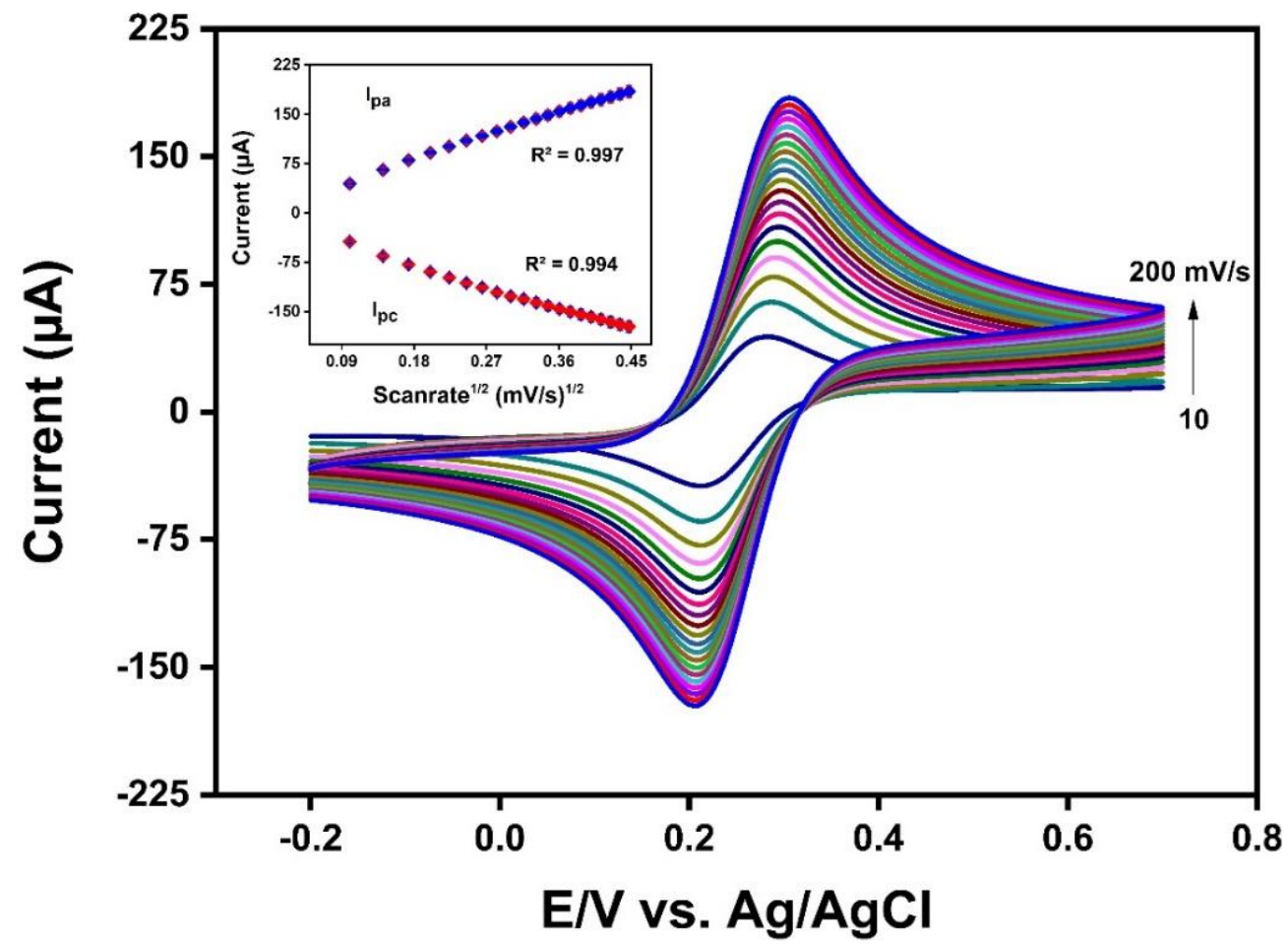

Fig. S2 CV curves for different scan rates from 10 to $200 \mathrm{mV} / \mathrm{s}$ at the TiC/BN/GCE in $5 \mathrm{mM}$ $\left[\mathrm{Fe}(\mathrm{CN})_{6}\right]^{3-14}$ containing $0.1 \mathrm{M} \mathrm{KCl}$ (Inset: calibration curves of square root of scan rate vs. peak current). 